\title{
Mechanism of influence of PR interval on loudness of first heart sound
}

\author{
GRAHAM LEECH, NICHOLAS BROOKS, ANNE GREEN-WILKINSON, \\ AUBREY LEATHAM
}

From the Cardiac Department, St. George's Hospital, Hyde Park Corner, London

SUMMARY It is well known that the loudness of the first heart sound is influenced by the duration of the PR interval and the position of the atrioventricular valve cusps at the moment of ventricular contraction, but the exact mechanism of this phenomenon has not been explained satisfactorily.

In this study, high speed echophonocardiograms were used to correlate the timing of mitral valve closure with the electrocardiogram and heart sounds in 67 normal subjects. Measurements were made of the PR interval and of the delay between the onset of ventricular depolarisation and mitral valve closure (Q-MC). To permit comparison of sound intensity between individuals, the amplitude of the mitral component of the first sound (M1) was compared with that of the aortic component of the second sound (A2) in the same subject.

When the PR interval was very long, mitral valve closure occurred soon after the onset of the electrocardiogram QRS complex, and there was no accompanying sound. Since the Q-MC interval in these cases was less than the electromechanical interval of $40 \mathrm{~ms}$, the valve was already closed before the onset of ventricular contraction and closure must have been effected by atrial contraction and relaxation. With PR intervals of less than $180 \mathrm{~ms}$ mitral closure was audible as a sharp sound coinciding with echocardiographic closure and occurring after the onset of ventricular contraction as judged by a Q-MC of $40 \mathrm{~ms}$ or more; the shorter the PR interval the later and louder the mitral closure sound. Thus a primary determinant of the intensity of $M 1$ is the timing of mitral closure in relation to the left ventricular pressure pulse. A full length PR interval gives time for coaptation of the mitral leaflets and closure occurs early as soon as left ventricular pressure starts to rise and the resulting sound is soft. A shorter PR interval, however, results in a wide open valve at the moment of ventricular contraction and hence delay in reaching its final closing halt which, therefore, occurs on a later and steeper part of the left ventricular pressure pulse and the sound is loud.

The effect of PR interval on the loudness. of the first heart sound was first described in atrioventricular block ${ }^{12}$; its importance as a major influence on the character of the sound in patients with conducted sinus rhythm was appreciated some time later. ${ }^{34}$ It is widely acknowledged that the effect is mediated through the partial atrioventricular valve closure induced by atrial contraction and relaxation, so that with a short PR interval the valve leaflets are widely separated, and with a long PR interval they are nearly apposed, when ventricular contraction begins. ${ }^{56}$ When one considers the small mass of the valve leaflets, however, it is difficult to understand why distance of travel alone, with implied variation in closing velocity, Received for publication 9 March 1979 should cause such great variations in loudness. It has been suggested that the critical factor is the timing of valve closure in relation to the ventricular pressure pulse, ${ }^{78}$ but correlation between this timing and the loudness of the first sound has not previously been demonstrated in man. There are a number of possible explanations for the difficulty in establishing such a relation. Because of the differing sound transmission properties of the lungs and chest wall, direct comparison of phonocardiograms between one person and another is not meaningful. In addition, alterations in the time between the onset of ventricular contraction (or electrical depolarisation) and valve closure are likely to be small and detectable only by sensitive techniques. Finally, it is frequently difficult to 
identify separately the mitral and tricuspid contributions to the first sound on conventional phonocardiograms. ${ }^{9}$

The present study has used high-speed simultaneous recordings of the echo and phonocardiogram to study the effect of atrioventricular conduction time on the timing of mitral valve closure and its corresponding component of the first heart sound (M1) in normal subjects. An attempt to overcome the problem of calibrating the sound recording has been made by comparing the amplitude of M1 with that of the aortic component of the second sound, A2.

\section{Methods}

Sixty-seven normal subjects were studied. Their ages ranged from 19 to 56 (mean 32) years and there were 27 men and 40 women.

The three standard bipolar limb leads of the electrocardiogram were recorded at $100 \mathrm{~mm} / \mathrm{s}$ paper speed and at four times normal calibration $(1 \mathrm{mV}=40 \mathrm{~mm})$. The lead showing the earliest QRS deflection was selected for recording with the echocardiogram and phonocardiogram.

Echocardiograms were recorded with a SmithKline Ekoline 20 ultrasonoscope $(2 \cdot 25 \mathrm{MHz}$ focused transducer, pulse repetition frequency $1850 \mathrm{~s}^{-1}$ ) interfaced with a Cambridge six-channel photographic recorder which had been modified to double echo depth magnification and with the maximum recording speed increased to $200 \mathrm{~mm} / \mathrm{s}$. The mitral valve was identified by the standard technique, and transducer angulation and position were adjusted to demonstrate the moment of valve closure, defined as the point of complete apposition of its anterior and posterior leaflets.

A high frequency phonocardiogram was recorded simultaneously with the echocardiogram using a Cambridge crystal microphone attached to the skin by suction. The microphone was placed accurately over the apex beat and the sensitivity of the amplifier was adjusted so that whenever possible both the first and second heart sounds could be recorded. The recordings were made in shallow held expiration, with maximal magnification of the echo display and at a film speed of $200 \mathrm{~mm} / \mathrm{s}$.

The PR interval was measured on the simultaneous standard lead electrocardiogram recording from the earliest $P$ wave onset to the earliest deflection of the QRS complex. The mean value was determined from five cardiac cycles measured to the nearest $0.5 \mathrm{~mm}(5 \mathrm{~ms})$. The time from the electrocardiogram $Q$ wave to mitral valve closure (Q-MC) was measured on the echocardiogram to the nearest $0.5 \mathrm{~mm}(2.5 \mathrm{~ms})$ using a hair-line cursor.
Five cardiac cycles were measured and the average value expressed to the nearest millisecond. The mitral component of the first sound (M1) was identified on the phonocardiogram from the exact coincidence of its onset with the earliest point of coaptation of the anterior and posterior cusps of the mitral valve on the echogram (Fig. 4). The peak-topeak amplitude of the maximum trace deflection was measured and from this the width of the baseline $(2.5 \mathrm{~mm})$ was subtracted. The maximum amplitude of the aortic component of the second sound (A2) was determined in the same manner. From these measurements the M1/A2 amplitude ratio was determined from the mean of five consecutive cycles.

\section{Results}

The mitral valve echogram was suitable for analysis in every case. Satisfactory phonocardiograms were recorded in 58 subjects.

In six subjects, all with PR intervals equal to or exceeding $180 \mathrm{~ms}$, no sound occurred at the time of mitral valve closure. In all the others valve closure was synchronous with the onset of the sound.

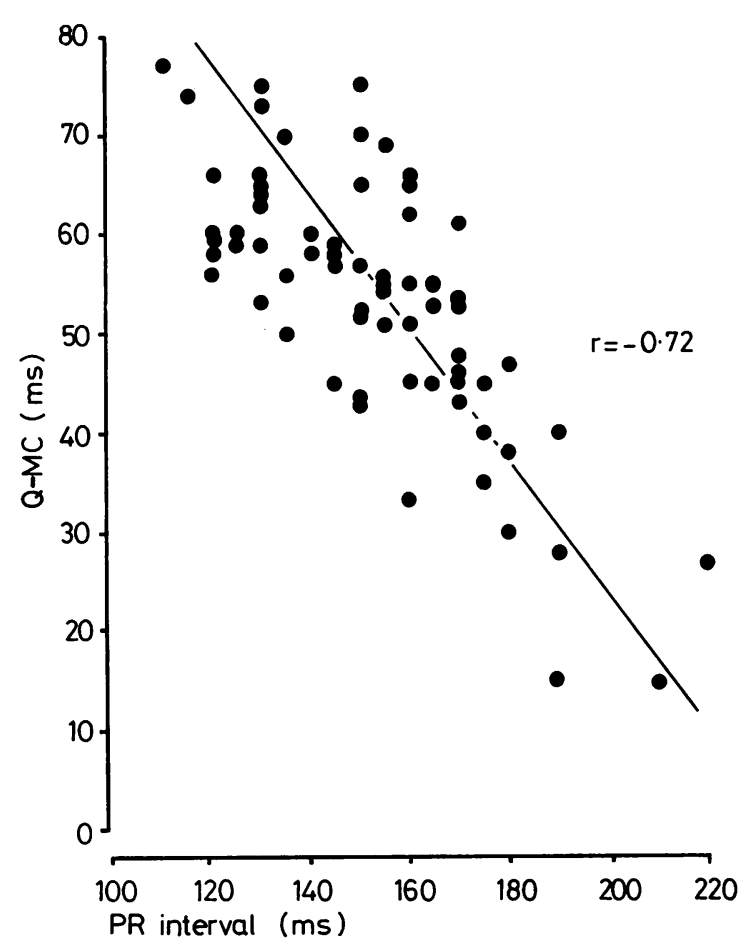

Fig. 1 The influence of $P R$ interval on the timing of mitral valve closure. 


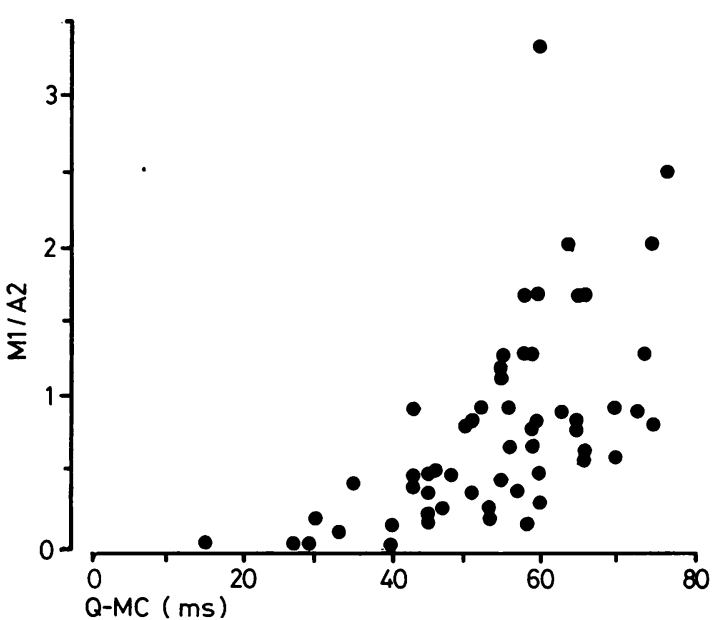

Fig. 2 The relation between the amplitude ratio $M 1 / A 2$ and the timing of mitral valve closure (Q-MC).

The influence of PR interval on the timing of mitral valve closure and $M 1$ is described in detail elsewhere. ${ }^{9}$ When the PR interval was long the valve leaflets were almost apposed at the time of ventricular depolarisation and Q-MC was short. With short PR intervals the valve was still widely open at the onset of the QRS complex and Q-MC was long. This relation between $P R$ interval and Q-MC is shown in Fig. 1, from which it can be seen that for a PR interval of 180 to $190 \mathrm{~ms}$ Q-MC is about $40 \mathrm{~ms}$, which is approximately the time between the initiation of depolarisation and the onset of left ventricular contraction. ${ }^{10} 11$ Thus, with PR intervals of $180 \mathrm{~ms}$ or greater, mitral valve closure occurs before the onset of ventricular contraction and is brought about entirely by atrial contraction and relaxation, while with shorter conduction times closure is completed after the beginning of ventricular contraction.

The relation between $\mathrm{Q}-\mathrm{MC}$ and the ratio $\mathrm{M1} / \mathrm{A} 2$ is shown in Fig. 2. Early mitral closure resulting from a long PR interval was associated with a small ratio and late valve closure in the presence of a short PR interval with a large ratio. When Q-MC fell to less than about $35 \mathrm{~ms}$ no $\mathrm{Ml}$ was recorded (that is mitral closure was silent) and the ratio was zero. Fig. 3 shows the recordings from two subjects aligned on the $Q$ wave of the electrocardiogram to illustrate these findings. In the first trace the PR interval is $110 \mathrm{~ms}, \mathrm{Q}-\mathrm{MC}$ is $77 \mathrm{~ms}$, and a large amplitude $M 1$ is recorded. In the second recording from a subject with a PR interval of $170 \mathrm{~ms}$, Q-MC is $48 \mathrm{~ms}$ and $M 1$ has a very small amplitude. With M1/A2 expressed as a logarithm the relation of $\log \mathrm{M} 1 / \mathrm{A} 2$ with Q-MC was examined by linear regression analysis. The correlation was highly significant $(r=0.65 ; p<0.001)$, and is shown in Fig. 4.

In Fig. 3, the closing velocity of the anterior mitral valve leaflet is obviously faster in the subject with a short PR interval and a loud first sound than in the individual with a long PR interval and a soft first sound, but there was no overall correlation between closing velocity and M1/A2 ratio. This is slightly surprising because such a relation has been observed in individual patients with complete heart block. ${ }^{12}$ However, since the closing mitral valve inscribes a curved line it is quite difficult to determine its velocity immediately before the point of complete closure, and we suspect that closing velocity is related to first sound amplitude, even though we have not been able to show it.

\section{Discussion}

By using a high recording speed we have been able to detect small differences in the timing of mitral

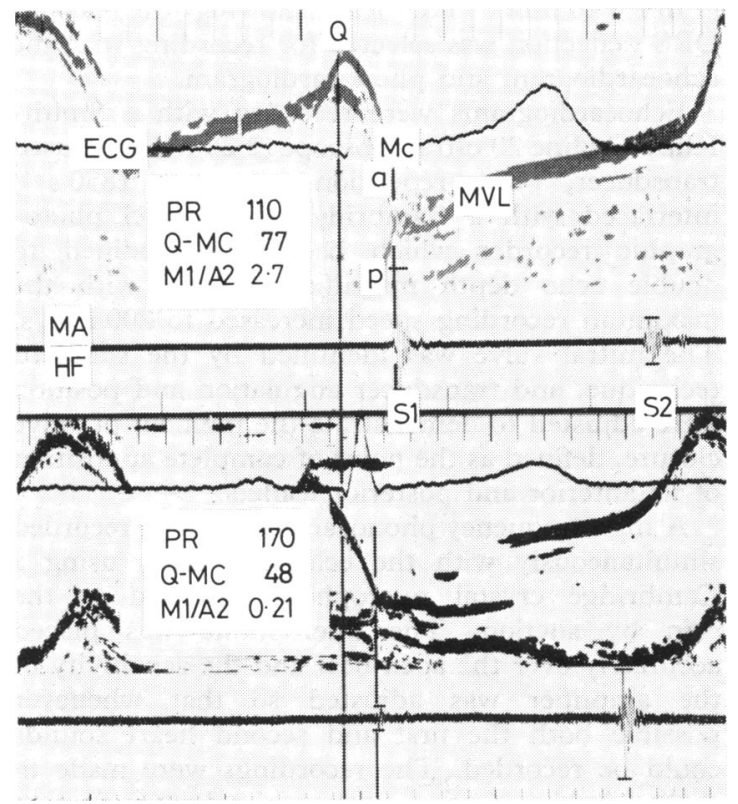

Fig. 3 Echophonocardiograms from two subjects aligned on the $Q$ waves of the electrocardiogram (ECG) to illustrate the influence of $P R$ interval on the timing of mitral valve closure and the amplitude of $M 1$ relative to A2 (see text). The onset of the $P$ wave is not clearly defined on these recordings; $P R$ interval was measured on the separate simultaneous recording of the three standard leads.

$a$, anterior cusp of mitral valve; $p$, posterior cusp of mitral valve. The maximum sound deflections are marked by short horizontal bars. 


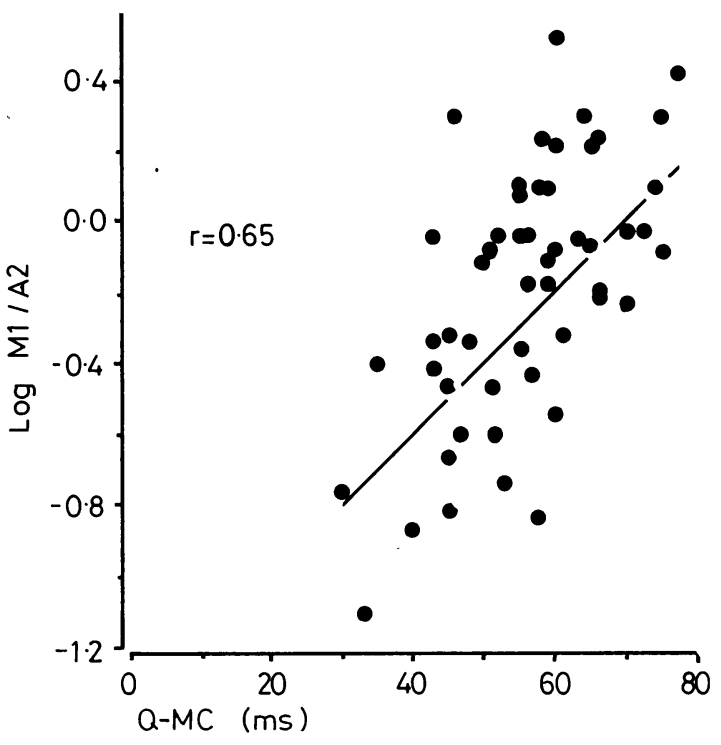

Fig. 4 The relation between $\log M 1 / A 2$ and the timing of the mitral valve closure ( $Q-M C)$.

valve closure and its corresponding component of the first heart sound (M1) associated with variations in the PR interval on the electrocardiogram. Use of the echo to identify $M 1$ is essential because under certain circumstances the first sound may be produced solely by tricuspid valve closure, mitral closure being silent. ${ }^{6} 9$ Furthermore, the method allows differentiation of $\mathrm{M} 1$ from the low-frequency vibrations which commonly precede it.

The major difficulty in studying any particular influence on the amplitude of heart sounds is the wide variation in anatomical factors which affect sound transmission from the site of production to the surface of the chest. We have attempted to overcome the problem by comparing the amplitude of $\mathrm{Ml}$ with that of the aortic component of the second sound (A2) at a fixed point on the praecordium, since both are affected by the same influences during their transmission to the surface. The validity of the study clearly rests on the assumption that the sound energy of $\mathrm{A} 2$ is constant in a group of resting normal subjects. The loudness of $A 2$ is known to be influenced by arterial pressure, the rate of pressure fall in late systole, and the physical properties of the aortic valve and $\operatorname{root}^{13}$; it was thought to be a fair assumption that these factors were 'normal' in our subjects though there must be some degree of biological variation. On this premise the energy of M1 was estimated by measuring the ratio of its amplitude compared with that of $\mathrm{A} 2$, with the microphone placed over the apex beat. An immediate criticism is that variations in the loudness of M1 may be associated with changes in its frequency, an alteration which would, itself affect transmission through the body tissues. ${ }^{13}$ Furthermore, the different frequency spectra of $M 1$ and A2 result in different degrees of attenuation during their transmission to the body surface. However, these criticisms do not invalidate our results, since variations in the amplitudes of A2 and $M 1$ unrelated to $P R$ interval would tend to scatter the results, rather than improve the correlation we have shown. Clearly, the method cannot be applied if the aortic valve is diseased or if left ventricular ejection dynamics are altered by peripheral, myocardial, or conduction abnormalities.

The effect of PR interval on the timing of mitral valve closure has been discussed elsewhere ${ }^{9}$; our observations support the conclusions of others, namely that when the PR interval is longer than 180 to $200 \mathrm{~ms}$, valve closure is brought about entirely by atrial activity and before the left ventricle starts to contract. ${ }^{5612}$ In the present investigation with a PR interval of 180 to $190 \mathrm{~ms}$, when presystolic valve closure was complete, the M1/A2 ratio fell to zero because of the total disappearance of M1. With shorter PR intervals valve closure occurred later in relation to the onset of QRS (and, therefore, later in relation to the onset of left ventricular contraction), and was associated with the production of a sound: the shorter the PR interval, the later valve closure, and the louder the sound. This concept of loudness being associated with lateness, widely accepted for mitral stenosis, ${ }^{14}$ has now been shown to be true also in normal subjects. We have confirmed the fact that closure of the mitral valve from the wide open position produces a loud sound and from a semiclosed position a soft sound. ${ }^{5} 6$ These differences are not directly the result of distance of travel of the cusps (always difficult to understand), but of varying delay related to distance of travel. This is in agreement with the concept put forward by McKusick ${ }^{15}$ and supported by Stept et al. ${ }^{8}$ that the critical factor determining the loudness of the sound is the change in momentum as blood being accelerated towards the left atrium is suddenly checked by the valve closure. The momentum of the blood is determined by the force acting on it (ventricular pressure) and the time over which the force acts. Thus the wider the separation of cusps the greater the delay in coming to a final halt and the louder the sound. We believe that it is the delay consequent upon the distance of travel which causes the final halt of the valve to be later, at a point where the rate of rise of the ventricular pressure pulse is steeper, and it is therefore the delay which is 
responsible for the increase in intensity of the first sound associated with a short PR interval.

GL and NB were supported by British Heart Foundation Research Fellowships.

The work described is part of a study submitted by NB to the University of London for the degree of Doctor of Medicine.

\section{References}

${ }^{1}$ Griffith TW. Remarks on two cases of heart block. Heart 1912; 3: 143-60.

${ }^{2}$ Lewis T. Lectures on the heart. New York: PB Hoeber, 1915.

${ }^{3}$ Wolferth CC, Margolies A. The influence of auricular contraction on the first heart sound and the radial pulse. Arch Intern Med 1930; 46: 1048-71.

${ }^{4}$ Levine SA, Harvey WP. Clinical auscultation of the heart. Philadelphia: W B Saunders, 1949.

${ }^{5}$ Zaky A, Steinmetz E, Feigenbaum H. Role of atrium in closure of the mitral valve in man. Am $\mathcal{F}$ Physiol 1969; 217: 1652-9.

${ }^{6}$ Burggraf GW, Craige E. The first heart sound in complete heart block. Phono-echocardiographic correlations. Circulation 1974; 50: 17-24.

${ }^{7}$ Little RC, Hilton JG, Schaefer RD. The first heart sound in normal and ventricular ectopic contractions.
Mechanism of closure of the A-V valves. Circ Res 1954; 2: 48-52.

${ }^{8}$ Stept ME, Heid CE, Shaver JA, Leon DF, Leonard JJ. Effect of altering $P-R$ interval on the amplitude of the first sound in the anesthetized dog. Cir Res 1969; 25: 255-63.

${ }^{9}$ Brooks N, Leech G, Leatham A. Factors responsible for normal splitting of first heart sound. High-speed echophonocardiographic study of valve movement. Br Heart F 1979; 42: 695-702.

${ }^{10}$ Braunwald E, Fishman AP, Cournand A. Time relationship of dynamic events in the cardiac chambers, pulmonary artery and aorta in man. Circ Res 1956; 4: 100-7.

${ }^{11}$ Luisada AA, Cortis B. The dynamic events of the normal heart in man. Acta Cardiol (Brux) 1970; 25: 203-12.

${ }^{12}$ Shah PM, Kramer DH, Gramiak R. Influence of the timing of atrial systole on mitral valve closure and on the first heart sound in man. Am $\mathcal{F}$ Cardiol 1970; 26: 231-7.

${ }^{13}$ Ravin A. Auscultation of the heart. Chicago: Yearbook Medical Publishers, 1967.

${ }^{14}$ Weiss O, Joachim G. Registrierung von Herztönen und Herzgeräuschen mittels des Phonoskops und ihre Beziehungen zum Elektrokardiogramm. Z. klin Med 1911; 73: 240-56.

${ }^{15}$ McKusick VA. Cardiovascular sound in health and disease. Baltimore: Williams \& Wilkins, 1958.

Requests for reprints to Dr Nicholas Brooks, Cardiac Department, The London Chest Hospital, Bonner Road, London E2 9JX. 